\title{
Globally Exponential Stability of Periodic Solutions to Impulsive Neural Networks with Time-Varying Delays
}

\author{
Yuanfu Shao, ${ }^{1}$ Changjin $\mathrm{Xu}{ }^{2}$ and Qianhong Zhang ${ }^{2}$ \\ ${ }^{1}$ College of Science, Guilin University of Technology, Guangxi, Guilin 541004, China \\ ${ }^{2}$ Guizhou Key Laboratory of Economics System, Guizhou College of Finance and Economics, \\ Guizhou, Guiyang 550004, China
}

Correspondence should be addressed to Yuanfu Shao, shaoyuanfu@163.com

Received 12 January 2012; Accepted 26 February 2012

Academic Editor: Josef Diblík

Copyright (c) 2012 Yuanfu Shao et al. This is an open access article distributed under the Creative Commons Attribution License, which permits unrestricted use, distribution, and reproduction in any medium, provided the original work is properly cited.

By using Schaeffer's theorem and Lyapunov functional, sufficient conditions of the existence and globally exponential stability of positive periodic solution to an impulsive neural network with time-varying delays are established. Applications, examples, and numerical analysis are given to illustrate the effectiveness of the main results.

\section{Introduction}

It is well known that in implementation of neural networks, time delays are inevitably encountered because of the finite switching speed of amplifiers. Specially in electronic neural networks, delays are usually time-varying and often become sources of instability. So it is important to investigate the dynamics of neural networks with delays [1-7]. Recently, the study of the existence of periodic solutions of neural networks has received much attention. The common approaches are based on using Mawhin continuation theorem [1, 2, 8-10], Banach's fixed point theorem [11-13], fixed point theorem in a cone [14], Schaeffer's theorem $[15,16]$, and so on. On the other hand, studies on neural dynamical systems not only involve the existence of periodic solutions, but also involve other dynamical behaviors such as stability of periodic solutions, bifurcations, and chaos. In recent years, the stability of solutions of neural networks has attracted attention of many researchers and many nice 
results have been obtained [1-3, 5-13, 16-25]. For example, M. Tan and Y. Tan [1] considered the following neural network with variable coefficients and time-varying delays:

$$
\begin{array}{r}
x_{i}^{\prime}(t)=-c_{i}(t) x_{i}(t)+\sum_{j=1}^{n} a_{i j}(t) f_{j}\left(x_{j}(t)\right)+\sum_{j=1}^{n} b_{i j}(t) f_{j}\left(x_{j}\left(t-\tau_{i j}(t)\right)\right)+J_{i}(t), \\
i=1,2, \ldots, n .
\end{array}
$$

By using the Mawhin continuation theorem, they discussed the existence and globally exponential stability of periodic solutions.

However, in real world, many physical systems often undergo abrupt changes at certain moments due to instantaneous perturbations, which lead to impulsive effects. In fact, impulsive differential equation represents a more natural framework for mathematical modelling of many real world phenomena such as population dynamic and neural networks. The theory of impulsive differential equations is now being recognized to be richer than the corresponding theory of differential equations without impulse, and various kinds of impulsive differential equations have been extensively studied, see $[8-16,18,19,21-25]$ and references therein. Then, considering impulsive effects, it is necessary and interesting for us to study further the dynamics of system (1.1). Furthermore, as pointed by Gopalsamy and Sariyasa [4], it would be of great interest to study neural networks in periodic environment. On the other hand, to the best of our knowledge, few authors considered the existence of periodic solutions by using Schaeffer's theorem. Hence, in this paper, by using Schaeffer's theorem and Lyapunov functional, we aim to discuss the existence and exponential stability of periodic solutions to a class of impulsive neural networks with periodic coefficients and time-varying delays. The model is as follows:

$$
\begin{gathered}
x_{i}^{\prime}(t)=-c_{i}(t) x_{i}(t)+\sum_{j=1}^{n} a_{i j}(t) f_{j}\left(x_{j}(t)\right)+\sum_{j=1}^{n} b_{i j}(t) f_{j}\left(x_{j}\left(t-\tau_{i j}(t)\right)\right)+J_{i}(t), \quad t \neq t_{k}, \\
x_{i}\left(t^{+}\right)=\left(1+q_{i}^{k}\right) x_{i}(t), \quad t=t_{k},
\end{gathered}
$$

with initial conditions

$$
x_{i}(s)=\phi_{i}(s), \quad \phi_{i}(s) \in C\left([-\tau, 0], R^{n}\right), i=1,2, \ldots, n,
$$

where $x_{i}(t)$ corresponds to the state of the $i$ th unit, $c_{i}(t)$ represents the rate with which the $i$ th unit will reset its potential to the resting state in isolation when disconnected from the network and external inputs, $f_{j}\left(x_{j}(t)\right)$ denotes the output of the $j$ th unit, $a_{i j}(t)$ and $b_{i j}(t)$ denote the strength of the $j$ th unit on the $i$ th unit, respectively, $J_{i}(t)$ is the external bias on the $i$ th unit, $\tau_{i j}(t)$ corresponds to the transmission delay along the axon of the $j$ th unit, $t_{k}$ denotes the impulsive moment, and $t_{1}<t_{2}<\cdots$ is a strictly increasing sequence such that $\lim _{k \rightarrow \infty} t_{k}=\infty, C\left([-\tau, 0], R^{n}\right)$ denotes the Banach space of continuous mapping from $[-\tau, 0]$ to $R^{n}$ equipped with the norm $\|\phi\|=\max _{1 \leq i \leq n} \sup _{t \in[0, \omega]}\left|\phi_{i}(t)\right|$ for all $\phi=\left(\phi_{1}(t), \phi_{2}(t), \ldots, \phi_{n}(t)\right)^{T} \in C\left([-\tau, 0], R^{n}\right)$, where $\tau=\max _{1 \leq i \leq n} \sup _{t \in[0, \omega]} \tau_{i j}(t)$. 
Throughout this paper, we always assume the following.

$\left(H_{1}\right) c_{i}(t)>0, a_{i j}(t), b_{i j}(t), J_{i}(t), \tau_{i j}(t)$ are all continuous $\omega$-periodic functions for $i, j=$ $1,2, \ldots, n$.

$\left(H_{2}\right) f_{j}: R \rightarrow R$ is continuous and there exists positive constant $k_{j}$ such that $f_{j}(u)-$ $f_{j}(v) \leq k_{j}|u-v|$ for any $u, v \in R$ and $j=1,2, \ldots, n$.

$\left(H_{3}\right)$ There exists positive integer $p$ such that $t_{k+p}=t_{k}+\omega, q_{i}^{k}=q_{i}^{k+p}$. Then

$$
[0, \omega] \cap\left\{t_{k}, k=1,2, \ldots\right\}=\left\{t_{1}, t_{2}, \ldots, t_{p}\right\}
$$

For convenience, we use the following notations:

$$
\bar{f}=\frac{1}{\omega} \int_{0}^{\omega}|f(t)| d t, \quad f^{M}=\max _{t \in[0, \omega]}|f(t)|, \quad f^{m}=\min _{t \in[0, \omega]}|f(t)|,
$$

where $f(t)$ is continuous and $\omega$-periodic function.

The rest of this paper is organized as follows. In Section 2, by using Schaeffer's theorem, sufficient conditions of the existence of $\omega$-periodic solution to system (1.2) with initial conditions (1.3) are established. In Section 3, by using Lyapunov functional, we derive the conditions under which the periodic solution is globally exponentially stable. In Section 4, applications, illustrative examples, and simulations are given to show the effectiveness of the main results. Finally, some conclusions are drawn in Section 5.

\section{Existence of Periodic Solution}

First we make some preparations. As usual in the theory of impulsive differential equation, by a solution of model (1.2), it means the following.

(i) $x(t)=\left(x_{1}(t), x_{2}(t), \ldots x_{n}(t)\right)^{T} \in R^{n}, x_{i}(t)$ is piecewise continuous such that $x_{i}\left(t_{k}^{-}\right)=$ $x_{i}\left(t_{k}\right), x_{i}\left(t_{k}^{+}\right)$exists, and $x_{i}(t)$ is differentiable on $\left(t_{k-1}, t_{k}\right)$ for $i=1,2, \ldots, n, k=$ $1,2, \ldots$

(ii) $x_{i}(t)$ satisfies $(1.2)$ for $i=1,2, \ldots, n$.

Definition 2.1. The set $A$ is said to be quasi-equicontinuous in $[0, \omega]$ if for any $\epsilon>0$, there exists $\delta>0$ such that, if $x \in A, t \in Z, t^{\prime}, t^{\prime \prime} \in\left(t_{k-1}, t_{k}\right) \cap[0, \omega]$ and $\left|t^{\prime}-t^{\prime \prime}\right|<\delta$, then $\left|x\left(t^{\prime}\right)-x\left(t^{\prime \prime}\right)\right|<\epsilon$.

Lemma 2.2 (see [26, Compactness criterion]). The set $A \subset X$ is relatively compact if and only if

(i) $A$ is bounded, that is, $\|x\| \leq M$ for each $x \in A$ and some $M>0$,

(ii) $A$ is quasi-equicontinuous in $[0, \omega]$.

The following lemma is fundamental to our discussion. The method is similar to that of $[13,16]$, so the proof is omitted here. 
Lemma 2.3. $x(t)=\left(x_{1}(t), x_{2}(t), \ldots, x_{n}(t)\right)^{T}$ is an w-periodic solution of system (1.2) which is equivalent to $x(t)=\left(x_{1}(t), x_{2}(t), \ldots, x_{n}(t)\right)^{T}$ is an w-periodic solution of the following equation:

$$
\begin{aligned}
x_{i}(t)= & \int_{0}^{\omega} G_{i}(t, s)\left(\sum_{j=1}^{n} a_{i j}(s) f_{j}\left(x_{j}(s)\right)+\sum_{j=1}^{n} b_{i j}(s) f_{j}\left(x_{j}\left(s-\tau_{i j}(s)\right)\right)+J_{i}(s)\right) d s \\
& +\sum_{k=1}^{p} G_{i}\left(t, t_{k}\right) q_{i}^{k} x_{i}\left(t_{k}\right),
\end{aligned}
$$

where $G(t, s)=\left(G_{1}(t, s), G_{2}(t, s), \ldots, G_{n}(t, s)\right)^{T}$, and

$$
G_{i}(t, s)= \begin{cases}\frac{e^{\int_{0}^{\omega} c_{i}(u) d u-\int_{s}^{t} c_{i}(u) d u}}{e^{\int_{0}^{\omega} c_{i}(u) d u}-1}, & 0 \leq s \leq t \leq \omega, \\ \frac{e^{\int_{t}^{s} c_{i}(u) d u}}{e^{\int_{0}^{\omega} c_{i}(u) d u}-1}, & 0 \leq t \leq s \leq \omega .\end{cases}
$$

It is easy to show that $G_{i}(t+\omega, s+\omega)=G_{i}(t, s), G_{i}(t, t+\omega)-G_{i}(t, t)=1$ and

$$
\frac{1}{\sigma_{i}-1} \leq G_{i}(t, s) \leq \frac{\sigma_{i}}{\sigma_{i}-1}
$$

where $\sigma_{i}=e^{\int_{0}^{\omega} c_{i}(u) d u}$ and $i=1,2, \ldots, n$.

Lemma 2.4 (see [27, Schaeffer's theorem]). Let X be a normed space and $\phi: X \rightarrow X$ be a compact operator. Define

$$
H(\phi)=\{x \mid x \in X, x=\lambda \phi x, 0<\lambda<1\} .
$$

Then either

(i) set $H(\phi)$ is unbounded, or

(ii) operator $\phi$ has a fixed point in $X$.

In order to use Lemma 2.4, let

$\operatorname{PC}\left([0, \omega], R^{n}\right)$

$$
=\left\{x:[0, \omega] \longrightarrow R^{n} \mid \lim _{s \rightarrow t} x(s)=x(t), t \neq t_{k}, \lim _{t \rightarrow t_{k}^{-}} x(t)=x\left(t_{k}\right), \lim _{t \rightarrow t_{k}^{+}} x(t) \text { exists, } k=1,2, \ldots, p\right\},
$$

with the norm $\|x\|=\max _{1 \leq i \leq n} \sup _{t \in[0, \omega]}\left|x_{i}(t)\right|$, then $\operatorname{PC}\left([0, \omega], R^{n}\right)$ is a Banach space. 
Define a mapping $\phi: \mathrm{PC}\left([0, \omega], R^{n}\right) \rightarrow \mathrm{PC}\left([0, \omega], R^{n}\right)$ by $(\phi x)(t)=x(t)$, where $(\phi x)(t)=$ $\left((\phi x)_{1}(t),(\phi x)_{2}(t), \ldots,(\phi x)_{n}(t)\right)^{T}$ and

$$
\begin{aligned}
(\phi x)_{i}(t)= & \int_{t}^{t+\omega} G_{i}(t, s)\left(\sum_{j=1}^{n} a_{i j}(s) f_{j}\left(x_{j}(s)\right)+\sum_{j=1}^{n} b_{i j}(s) f_{j}\left(x_{j}\left(s-\tau_{i j}(s)\right)\right)+J_{i}(s)\right) d s \\
& +\sum_{k=1}^{p} G_{i}\left(t, t_{k}\right) q_{i}^{k} x_{i}\left(t_{k}\right) .
\end{aligned}
$$

By Lemma 2.3, it is easy to see that the existence of w-periodic solution of (1.2) is equivalent to the existence of fixed point of the mapping $\phi$ in $\mathrm{PC}\left([0, \omega], R^{n}\right)$.

Theorem 2.5. Suppose that $\left(H_{1}\right)-\left(H_{3}\right)$ hold. Further,

$$
\left(H_{4}\right) \max _{1 \leq i \leq n}\left(\sigma_{i} / \sigma_{i}-1\right)\left(\sum_{j=1}^{n}\left(\overline{a_{i j}}+\overline{b_{i j}}\right) k_{j}+\sum_{k=1}^{p}\left|q_{i}^{k}\right|\right):=\theta<1 .
$$

Then system (1.2) admits an w-periodic solution.

Proof. By Lemma 2.3, it suffices to prove that the mapping $\phi$ admits a fixed point in $\operatorname{PC}\left([0, \omega], R^{n}\right)$.

For any constant $H>0$, let $\Omega=\left\{x \mid x \in \mathrm{PC}\left([0, \omega], R^{n}\right),\|x\|<H\right\}$. For $x \in \Omega$, from (2.3) and $\left(H_{2}\right)$, we have

$$
\begin{aligned}
\|\phi x\|= & \max _{1 \leq i \leq n} \sup _{t \in[0, \omega]} \mid \int_{t}^{t+\omega} G_{i}(t, s)\left(\sum_{j=1}^{n} a_{i j}(s) f_{j}\left(x_{j}(s)\right)+\sum_{j=1}^{n} b_{i j}(s) f_{j}\left(x_{j}\left(s-\tau_{i j}(s)\right)\right)+J_{i}(s)\right) d s \\
& +\sum_{k=1}^{p} G_{i}\left(t, t_{k}\right) q_{i}^{k} x_{i}\left(t_{k}\right) \mid \\
\leq & \max _{1 \leq i \leq n} \sup _{t \in[0, \omega]} \frac{\sigma_{i}}{\sigma_{i}-1} \int_{0}^{\omega}\left(\sum_{j=1}^{n}\left|a_{i j}(t) f_{j}\left(x_{j}(t)\right)\right|+\sum_{j=1}^{n}\left|b_{i j}(t) f_{j}\left(x_{j}\left(t-\tau_{i j}(t)\right)\right)\right|+\left|J_{i}(t)\right|\right) d t \\
& +\frac{\sigma_{i}}{\sigma_{i}-1} \sum_{k=1}^{p}\left|q_{i}^{k} x_{i}\left(t_{k}\right)\right| \\
\leq & \max _{1 \leq i \leq n} \sup _{t \in[0, \omega]} \frac{\sigma_{i}}{\sigma_{i}-1} \int_{0}^{\omega}\left(\sum_{j=1}^{n}\left|a_{i j}(t)\right|\left(k_{j}\left|x_{j}(t)\right|+\left|f_{j}(0)\right|\right)\right. \\
& +\int_{0}^{\omega}\left|J_{i}(t)\right| d t+\frac{\sigma_{i}}{\sigma_{i}-1} \sum_{k=1}^{p}\left|q_{i}^{k} x_{i}\left(t_{k}\right)\right|
\end{aligned}
$$




$$
\begin{aligned}
& \leq \max _{1 \leq i \leq n} \frac{\sigma_{i}}{\sigma_{i}-1}\left(\sum_{j=1}^{n}\left(\overline{a_{i j}}+\overline{b_{i j}}\right) k_{j}+\sum_{k=1}^{p}\left|q_{i}^{k}\right|\right)\|x\|+\frac{\sigma_{i}}{\sigma_{i}-1}\left(\overline{J_{i}}+\sum_{j=1}^{n}\left|f_{j}(0)\right|\left(\overline{a_{i j}}+\overline{b_{i j}}\right)\right) \\
& \leq \max _{1 \leq i \leq n} \frac{\sigma_{i}}{\sigma_{i}-1}\left(\sum_{j=1}^{n}\left(\overline{a_{i j}}+\overline{b_{i j}}\right) k_{j}+\sum_{k=1}^{p}\left|q_{i}^{k}\right|\right) H+\frac{\sigma_{i}}{\sigma_{i}-1}\left(\overline{J_{i}}+\sum_{j=1}^{n}\left|f_{j}(0)\right|\left(\overline{a_{i j}}+\overline{b_{i j}}\right)\right):=R .
\end{aligned}
$$

It implies that $\phi(\Omega)$ is uniformly bounded.

For any $t \in[0, \omega], x \in \Omega$, we have

$$
\begin{aligned}
(\phi x)_{i}^{\prime}(t)=\frac{d}{d t}\left(\int_{t}^{t+\omega} G_{i}(t, s)\left(\sum_{j=1}^{n} a_{i j}(s) f_{j}\left(x_{j}(s)\right)+\sum_{j=1}^{n} b_{i j}(t) f_{j}\left(x_{j}\left(s-\tau_{i j}(s)\right)\right)+J_{i}(s)\right) d s\right. \\
\left.\quad+\sum_{k=1}^{p} G_{i}\left(t, t_{k}\right) q_{i} x_{i}\left(t_{k}\right)\right) \\
=-c_{i}(t)(\phi x)_{i}(t)+\left(\sum_{j=1}^{n} a_{i j}(t) f_{j}\left(x_{j}(t)\right)+\sum_{j=1}^{n} b_{i j}(t) f_{j}\left(x_{j}\left(t-\tau_{i j}(t)\right)\right)+J_{i}(t)\right) .
\end{aligned}
$$

If $t=t_{k}$, it is obvious that $(\phi x)_{i}^{\prime}(t)=\lim _{t \rightarrow t_{k}^{-}}(\phi x)_{i}^{\prime}(t)$. Hence, from (2.7) and (2.8), we have

$$
\left|(\phi x)_{i}^{\prime}(t)\right| \leq c_{i}^{M} R+\sum_{j=1}^{n}\left(\left(a_{i j}^{M}+b_{i j}^{M}\right) k_{j}\right) H+\left(J_{i}^{M}+\sum_{j=1}^{n}\left|f_{j}(0)\right|\left(a_{i j}^{M}+b_{i j}^{M}\right)\right):=\beta_{i} .
$$

Therefore, $\phi(\Omega) \subset \operatorname{PC}\left([0, \omega], R^{n}\right)$ is a family of uniformly bounded and equicontinuous subset. By Lemma 2.2, the mapping $\phi$ is compact.

Let $x \in \mathrm{PC}\left([0, \omega], R^{n}\right)$, and considering the following operator equation:

$$
x=\lambda(\phi x), \quad \lambda \in(0,1) .
$$

If $x$ is a solution of (2.10), then

$$
\|x\| \leq\|\phi x\| \leq \theta\|x\|+\max _{1 \leq i \leq n} \frac{\sigma_{i}}{\sigma_{i}-1}\left(\overline{J_{i}}+\sum_{j=1}^{n}\left|f_{j}(0)\right|\left(\overline{a_{i j}}+\overline{b_{i j}}\right)\right) .
$$

According to $\left(H_{4}\right)$, we deduce that

$$
\|x\| \leq \frac{\max _{1 \leq i \leq n}\left(\sigma_{i} /\left(\sigma_{i}-1\right)\right)\left(\overline{J_{i}}+\sum_{j=1}^{n}\left|f_{j}(0)\right|\left(\overline{a_{i j}}+\overline{b_{i j}}\right)\right)}{1-\theta}:=M
$$


It implies that $\|x\|$ is bounded, which is independent of $\lambda \in(0,1)$. By Lemma 2.4, we obtain that the mapping $\phi$ admits a fixed point in $\mathrm{PC}\left([0, \omega], R^{n}\right)$. Hence system (1.2) admits an $\omega$ periodic solution such that $\|x\| \leq M$. This completes the proof.

\section{Globally Exponentially Stable}

In this section, the sufficient conditions ensuring that (1.2) admits a unique $\omega$-periodic solution and all solutions of (1.2) exponentially converge to the unique $\omega$-periodic solution are to be established.

Definition 3.1. Let $x^{*}(t)$ be an $\omega$-periodic solution of system (1.2) with initial value $\phi^{*}$. If there exist constants $\alpha>0, P \geq 1$, for every solution $x(t)$ of (1.2) with initial $\phi$, such that

$$
\left\|x_{i}(t)-x_{i}^{*}(t)\right\| \leq P\left\|\phi-\phi^{*}\right\| e^{-\alpha t} \quad \text { for any } t>0, i=1,2, \ldots, n,
$$

then $x^{*}(t)$ is said to be globally exponentially stable.

Theorem 3.2. Suppose that $\left(H_{1}\right)-\left(H_{4}\right)$ hold. Further,

$$
\begin{aligned}
& \left(H_{5}\right)-c_{i}^{m}+\sum_{j=1}^{n}\left(a_{i j}^{M}+b_{i j}^{M}\right) k_{j}<0, \quad i=1,2, \ldots, n, \\
& \left(H_{6}\right) \ln \max _{1 \leq i \leq n}\left|1+q_{i}^{k}\right| / t_{k}-t_{k-1} \leq \zeta<\alpha,
\end{aligned}
$$

where $\max _{1 \leq i \leq n}\left|1+q_{i}^{k}\right| \geq 1, \zeta>0$ is a constant, $\alpha$ is a constant determined in (3.5).

Then system (1.2) admits a unique w-periodic solution, which is globally exponentially stable.

Proof. By Theorem 2.5, system (1.2) admits an $\omega$-periodic solution $x^{*}(t)=\left(x_{1}^{*}(t), x_{2}^{*}(t), \ldots\right.$, $\left.x_{n}^{*}(t)\right)$ with initial value $\phi^{*}$. Let $x(t)=\left(x_{1}(t), x_{2}(t), \ldots, x_{n}(t)\right)$ be an arbitrary solution of (1.2) with initial value $\phi$. Define $z_{i}(t)=x_{i}^{*}(t)-x_{i}(t)$ and $g_{j}\left(z_{j}(t)\right)=f_{j}\left(z_{j}(t)+x_{j}(t)\right)-f_{j}\left(x_{j}(t)\right)$, then we have

$$
\begin{gathered}
z_{i}^{\prime}(t)=-c_{i}(t) z_{i}(t)+\sum_{j=1}^{n} a_{i j}(t) g_{j}\left(z_{j}(t)\right)+\sum_{j=1}^{n} b_{i j}(t) g_{j}\left(z_{j}\left(t-\tau_{i j}(t)\right)\right), \quad t \neq t_{k}, \\
z_{i}\left(t^{+}\right)=\left(1+q_{i}^{k}\right) z_{i}(t), \quad t=t_{k} .
\end{gathered}
$$

By $\left(H_{5}\right)$, we have $-c_{i}^{m}+\sum_{j=1}^{n}\left(a_{i j}^{M}+b_{i j}^{M}\right) k_{j}<0$ for $i=1,2, \ldots, n$. Let

$$
h_{i}(\lambda)=\lambda-c_{i}^{m}+e^{\lambda \tau} \sum_{j=1}^{n}\left(a_{i j}^{M}+b_{i j}^{M}\right) k_{j}
$$

It is clear that $h_{i}(\lambda)$ is continuous on $R$ and $h_{i}(0)<0, i=1,2, \ldots, n$. In addition,

$$
\frac{d\left(h_{i}(\lambda)\right)}{d \lambda}=1+\tau e^{\lambda \tau} \sum_{j=1}^{n}\left(a_{i j}^{M}+b_{i j}^{M}\right) k_{j}>0,
$$


and $h_{i}(+\infty)=+\infty$, then $h_{i}(\lambda)$ is strictly monotone increasing. Therefore, there exists a unique $\lambda_{i}>0$ such that $\lambda_{i}-c_{i}^{m}+e^{\lambda_{i} \tau} \sum_{j=1}^{n}\left(a_{i j}^{M}+b_{i j}^{M}\right) k_{j}=0$ for $i=1,2, \ldots, n$. Let

$$
\alpha=\min \left\{\lambda_{1}, \lambda_{2}, \ldots, \lambda_{n}\right\},
$$

then

$$
h_{i}(\alpha)=\alpha-c_{i}^{m}+e^{\alpha \tau} \sum_{j=1}^{n}\left(a_{i j}^{M}+b_{i j}^{M}\right) k_{j} \leq 0, \quad i=1,2, \ldots, n .
$$

Obviously, for $t \in[-\tau, 0]$ and the above $\alpha$, we have

$$
\left|z_{i}(t)\right| \leq\left\|\phi-\phi^{*}\right\| \leq\left\|\phi-\phi^{*}\right\| e^{-\alpha t}, \quad i=1,2, \ldots, n,
$$

where $\left\|\phi-\phi^{*}\right\|=\max _{1 \leq i \leq n} \sup _{-\tau \leq s \leq 0}\left|\phi_{i}(s)-\phi_{i}^{*}(s)\right|$.

Define $V(t)=\left(V_{1}(t), V_{2}(t), \ldots, V_{n}(t)\right)^{T}$ by

$$
V_{i}(t)=e^{\alpha t}\left|z_{i}(t)\right|, \quad i=1,2, \ldots, n
$$

In view of (3.2) and (3.8), for $t \neq t_{k}$, we have

$$
\begin{aligned}
\frac{d^{+} V_{i}(t)}{d t} & =\alpha e^{\alpha t}\left|z_{i}(t)\right|+e^{\alpha t} \operatorname{sgn} z_{i}(t)\left\{-c_{i}(t) z_{i}(t)+\sum_{j=1}^{n} a_{i j}(t) g_{j}\left(z_{j}(t)\right)+\sum_{j=1}^{n} b_{i j}(t) g_{j}\left(z_{j}\left(t-\tau_{i j}(t)\right)\right)\right\} \\
& \leq\left(\alpha-c_{i}(t)\right) e^{\alpha t}\left|z_{i}(t)\right|+e^{\alpha t} \sum_{j=1}^{n}\left|a_{i j}(t)\right| k_{j}\left|z_{j}(t)\right|+e^{\alpha t} \sum_{j=1}^{n}\left|b_{i j}(t)\right| k_{j}\left|z_{j}\left(t-\tau_{i j}(t)\right)\right| \\
& \leq\left(\alpha-c_{i}^{m}\right) V_{i}(t)+\sum_{j=1}^{n} a_{i j}^{M} k_{j} V_{j}(t)+e^{\alpha \tau} \sum_{j=1}^{n} b_{i j}^{M} k_{j} V_{j}\left(t-\tau_{i j}(t)\right) .
\end{aligned}
$$

We claim that

$$
V_{i}(t)=e^{\alpha t}\left|z_{i}(t)\right| \leq\left\|\phi-\phi^{*}\right\| \quad \text { for } t \in\left(0, t_{1}\right), i=1,2, \ldots, n .
$$

If not, then there exist $i_{0} \in\{1,2, \ldots, n\}$ and $0<\bar{t}<t_{1}$ such that

$$
V_{i_{0}}(\bar{t})=\left\|\phi-\phi^{*}\right\|, \quad \frac{d^{+} V_{i_{0}}(\bar{t})}{d t}>0, \quad V_{i}(t) \leq\left\|\phi-\phi^{*}\right\|,
$$


for $t \in(-\tau, \bar{t}], i=1,2, \ldots, n$. Then, it follows from (3.9) and (3.11) that

$$
\begin{aligned}
0 & <\frac{d^{+} V_{i_{0}}(\bar{t})}{d t} \leq\left(\alpha-c_{i}^{m}\right) V_{i}(\bar{t})+\sum_{j=1}^{n} a_{i j}^{M} k_{j} V_{j}(\bar{t})+e^{\alpha \tau} \sum_{j=1}^{n} b_{i j}^{M} k_{j} V_{j}\left(\bar{t}-\tau_{i j}(\bar{t})\right) \\
& \leq\left(\alpha-c_{i_{0}}^{m}+e^{\alpha \tau} \sum_{j=1}^{n}\left(a_{i j}^{M}+b_{i j}^{M}\right) k_{j}\right)\left\|\phi-\phi^{*}\right\| .
\end{aligned}
$$

Equation (3.12) leads to

$$
\alpha-c_{i_{0}}^{m}+e^{\alpha \tau} \sum_{j=1}^{n}\left(a_{i_{0} j}^{M}+b_{i_{0} j}^{M}\right) k_{j}>0
$$

which contradicts (3.6). Thus (3.10) holds, that is,

$$
z_{i}(t) \leq\left\|\phi-\phi^{*}\right\| e^{-\alpha t}, \quad \text { for any } t \in\left[0, t_{1}\right), i=1,2, \ldots, n \text {. }
$$

If $t=t_{1}$, we have

$$
\left|z_{i}\left(t_{1}^{+}\right)\right|=\left|\left(1+q_{i}^{1}\right) z_{i}\left(t_{1}\right)\right|=\left|1+q_{i}^{1}\right| \lim _{t \rightarrow t_{1}^{-}}\left|z_{i}(t)\right| \leq\left|1+q_{i}^{1}\right|\left\|\phi-\phi^{*}\right\| e^{-\alpha t_{1}},
$$

for $i=1,2, \ldots, n$. Similar to the steps of (3.10)-(3.14), we can derive that

$$
\left|z_{i}(t)\right| \leq\left|1+q_{i}^{1}\right|\left\|\phi-\phi^{*}\right\| e^{-\alpha t}, \quad \text { for } t \in\left[t_{1}, t_{2}\right), i=1,2, \ldots, n .
$$

If $t=t_{2}$, then

$$
\left|z_{i}\left(t_{2}^{+}\right)\right|=\left|\left(1+q_{i}^{2}\right) z_{i}\left(t_{2}\right)\right| \leq\left|\left(1+q_{i}^{1}\right)\left(1+q_{i}^{2}\right)\right|\left\|\phi-\phi^{*}\right\| e^{-\alpha t_{2}}
$$

By repeating the same procedure, then

$$
\left|z_{i}(t)\right| \leq\left|\left(1+q_{i}^{1}\right)\left(1+q_{i}^{2}\right) \cdots\left(1+q_{i}^{p}\right)\right| \cdot\left\|\phi-\phi^{*}\right\| e^{-\alpha t}, \quad t \in\left(t_{p}, t_{p+1}\right), i=1,2, \ldots, n .
$$

It follows from $\left(H_{6}\right)$ that $\left|1+q_{i}^{k}\right| \leq e^{\zeta\left(t_{k}-t_{k-1}\right)}$, which leads to

$$
\left|\left(1+q_{i}^{1}\right)\left(1+q_{i}^{2}\right) \cdots\left(1+q_{i}^{p}\right)\right| \leq e^{\zeta\left(t_{1}-t_{0}\right)} e^{\zeta\left(t_{2}-t_{1}\right)} \cdots e^{\zeta\left(t_{p}-t_{p-1}\right)} \leq e^{\zeta t} e^{\zeta\left(\omega-t_{p}\right)},
$$

for any $t \in\left[t_{k}, t_{k+1}\right), i=1,2, \ldots, n, k=1,2, \ldots$ So the combination (3.18) and (3.19) gives

$$
\left|z_{i}(t)\right| \leq e^{\zeta\left(\omega-t_{p}\right)}\left\|\phi-\phi^{*}\right\| e^{-(\alpha-\zeta) t}, \quad t \in\left[t_{k}, t_{k+1}\right), i=1,2, \ldots, n, k=1,2, \ldots
$$


In addition, it is clear that

$$
\left|z_{i}(t)\right| \leq e^{\zeta\left(\omega-t_{p}\right)}\left\|\phi-\phi^{*}\right\| e^{-(\alpha-\zeta) t}, \quad t \in\left[0, t_{1}\right), i=1,2, \ldots, n .
$$

Therefore, from (3.20) and (3.21), for any $t>0$, we have

$$
\left|x_{i}(t)-x_{i}^{*}(t)\right|=\left|z_{i}(t)\right| \leq e^{\zeta\left(\omega-t_{p}\right)}\left\|\phi-\phi^{*}\right\| e^{-(\alpha-\zeta) t}, \quad i=1,2, \ldots, n .
$$

It implies that the $\omega$-periodic solution $x^{*}(t)$ of (1.2) is globally exponentially stable. Hence, (1.2) admits a unique $\omega$-periodic solution, which is globally exponentially stable. This completes the proof.

Remark 3.3. Theorem 3.2 implies that the impulse $q_{i}^{k}$ affects the existence and exponential stability of the periodic solution of system (1.2). It shows the dynamics of impulsive differential system (1.2) is richer than the corresponding system (1.1) without impulse.

\section{Applications and Examples}

In (1.2), if $a_{i j}(t) \equiv 0$, then (1.2) reads:

$$
\begin{gathered}
x_{i}^{\prime}(t)=-c_{i}(t) x_{i}(t)+\sum_{j=1}^{n} b_{i j}(t) f_{j}\left(x_{j}\left(t-\tau_{i j}(t)\right)\right)+J_{i}(t), \quad t \neq t_{k}, \\
x_{i}\left(t^{+}\right)=\left(1+q_{i}^{k}\right) x_{i}(t), \quad t=t_{k} .
\end{gathered}
$$

For system (4.1), we have the following result.

Proposition 4.1. Suppose that $\left(H_{1}\right)-\left(H_{3}\right)$ hold. Further,

$$
\begin{aligned}
& \left(H_{7}\right) \max _{1 \leq i \leq n}\left(\sigma_{i} /\left(\sigma_{i}-1\right)\right)\left(\sum_{j=1}^{n} \overline{b_{i j}} k_{j}+\sum_{k=1}^{p}\left|q_{i}^{k}\right|\right):=\theta<1, \\
& \left(H_{8}\right)-c_{i}^{m}+\sum_{j=1}^{n} b_{i j}^{M} k_{j}<0, \\
& \left(H_{9}\right) \ln \max _{1 \leq i \leq n}\left|1+q_{i}^{k}\right| /\left(t_{k}-t_{k-1}\right) \leq \zeta<\alpha,
\end{aligned}
$$

where $\max _{1 \leq i \leq n}\left|1+q_{i}^{k}\right| \geq 1, \zeta>0$ is a constant, $\alpha$ is determined in Theorem 3.2.

Then system (4.1) admits a unique w-periodic solution, which is globally exponentially stable.

If the impulses are absent in system (1.2), that is, $q_{i}^{k} \equiv 0$, then (1.2) leads to (1.1). Similarly we have the following.

Proposition 4.2. Suppose that $\left(H_{1}\right)-\left(H_{3}\right)$ hold. Further,

$$
\begin{aligned}
& \left(H_{10}\right) \max _{1 \leq i \leq n}\left(\sigma_{i} /\left(\sigma_{i}-1\right)\right) \sum_{j=1}^{n}\left(\overline{a_{i j}}+\overline{b_{i j}}\right) k_{j}:=\theta<1, \\
& \left(H_{11}\right)-c_{i}^{m}+\sum_{j=1}^{n}\left(a_{i j}^{M}+b_{i j}^{M}\right) k_{j}<0,
\end{aligned}
$$

then system (1.1) admits a unique w-periodic solution, which is globally exponentially stable. 
Remark 4.3. Proposition 4.2 implies that the sufficient conditions of the existence and globally exponential stability of periodic solution to (1.1) are independent of the time-varying delays, while the corresponding results obtained by authors [5] are dependent on delays. Without effect from time-varying delays, our results are better for people to keep the stability of system (1.1). Although the authors [1] also established similar conditions which are independent of delays, their employed tool and analysis techniques are very different so that their main results are different from ours. Particularly, (1.1) is the special case of (1.2) without impulse. Hence, in this sense, results of this paper complement or improve some previously known results $[1,5]$.

Finally, two examples and numerical analysis are given to show the usefulness of the main results.

Example 4.4. Let

$$
\begin{aligned}
& x_{1}^{\prime}(t)=-c_{1}(t) x_{1}(t)+b_{11}(t) f\left(x_{1}\left(t-\tau_{11}(t)\right)+b_{12}(t) f\left(x_{2}\left(t-\tau_{12}(t)\right)+J_{1}(t),\right.\right. \\
& x_{2}^{\prime}(t)=-c_{2}(t) x_{2}(t)+b_{21}(t) f\left(x_{1}\left(\mathrm{t}-\tau_{21}(t)\right)+b_{22}(t) f\left(x_{2}\left(t-\tau_{22}(t)\right)+J_{2}(t),\right.\right. \\
& \Delta x_{i}(t)=\left(1+q_{i}^{k}\right) x_{i}(t), \quad i=1,2,
\end{aligned}
$$

where $f(x)=x, c_{1}(t)=1+\sin \pi t / 4, c_{2}(t)=2+\cos \pi t / 4, a_{11}(t)=a_{12}(t)=a_{21}(t)=a_{22}(t)=$ $0, b_{11}(t)=1 / 8+\sin \pi t / 16, b_{12}(t)=1 / 8+\cos \pi t / 16, b_{21}(t)=1 / 16-\cos \pi t / 32, b_{22}(t)=1 / 16-$ $\sin \pi t / 24, \tau_{11}(t)=\tau_{12}(t)=\cos \pi t, \tau_{21}(t)=\tau_{22}(t)=1 / 2-\sin \pi t / 3, J_{1}(t)=5+2 \cos \pi t, J_{2}(t)=$ $7-\sin \pi t, q_{i}^{k}=1 / 8, t_{k}=k-1 / 2$. Then $k_{1}=k_{2}=1, \omega=2, \tau=1,\left\{t_{k}, k=1,2, \ldots\right\} \cap[0,2]=$ $\left\{t_{1}, t_{2}\right\}$.

By easy computation, $\sigma_{1}=e^{2}, \sigma_{2}=e^{4}$, and $\theta \approx 0.8674<1$, which implies $\left(H_{4}\right)$ holds. On the other hand, it is easy to verify that $\left(H_{5}\right)$ holds. By verification, $\alpha>1 / 4>\ln (1+(1 / 8))$, namely, $\left(H_{6}\right)$ holds too. From Theorems 2.5 and 3.2, we obtain that (4.2) has a unique 2periodic solution, which is globally exponentially stable, see Figure 1.

Example 4.5. Let

$$
\begin{aligned}
x_{1}^{\prime}(t)= & -\left(\frac{1}{2}+\frac{\sin 2 \pi t}{4}\right) x_{1}(t)+\left(\frac{1}{4}+\frac{\cos 2 \pi t}{6}\right) f\left(x_{1}(t-(2+\sin 2 \pi t))\right) \\
& +\left(\frac{1}{4}-\frac{\sin 2 \pi t}{8}\right) f\left(x_{2}(t-(3-\sin 2 \pi t))+\cos 2 \pi t\right), \\
x_{2}^{\prime}(t)= & -\left(\frac{1}{2}+\frac{\cos 2 \pi t}{4}\right) x_{2}(t)+\left(\frac{1}{3}+\frac{\cos 2 \pi t}{4}\right) f\left(x_{1}(t-(5-\sin 2 \pi t))\right) \\
& +\left(\frac{1}{6}-\frac{\cos 2 \pi t}{8}\right) f\left(x_{2}(t-(1+\cos 2 \pi t))+\sin 2 \pi t\right),
\end{aligned}
$$

where $f(x)=(1 / 4) x$ for $x \in R, c_{1}(t)=1 / 2+\sin 2 \pi t / 4, c_{2}(t)=1 / 2+\cos 2 \pi t / 4, a_{11}(t)=a_{12}(t)=$ $a_{21}(t)=a_{22}(t)=0, b_{11}(t)=1 / 4+\cos 2 \pi t / 6, b_{12}(t)=1 / 4-\sin 2 \pi t / 8, b_{21}(t)=1 / 3+\cos 2 \pi t / 4$, $b_{22}(t)=1 / 6-\cos 2 \pi t / 8$. Then $k_{1}=k_{2}=(1 / 4), \omega=1$.

By computation, $\theta \approx 0.318<1$, which implies that $\left(H_{10}\right)$ holds. It is easy to verify that $\left(H_{11}\right)$ holds too. From Proposition 4.2, system (4.3) has a unique 1-periodic solution, which is globally exponentially stable, see Figure 2 . However, by calculation, conditions of the results 


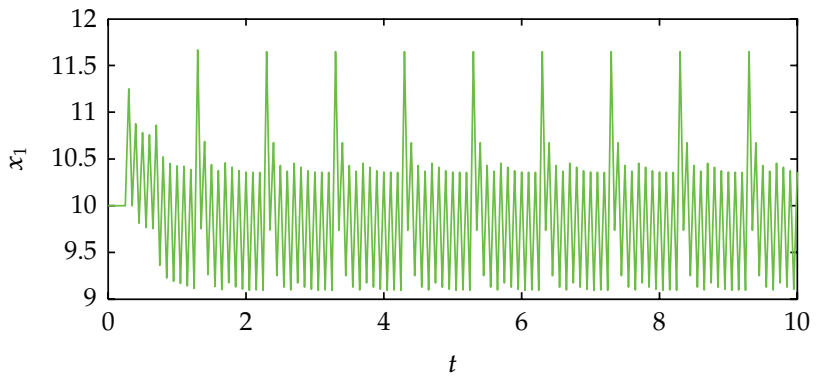

(a)

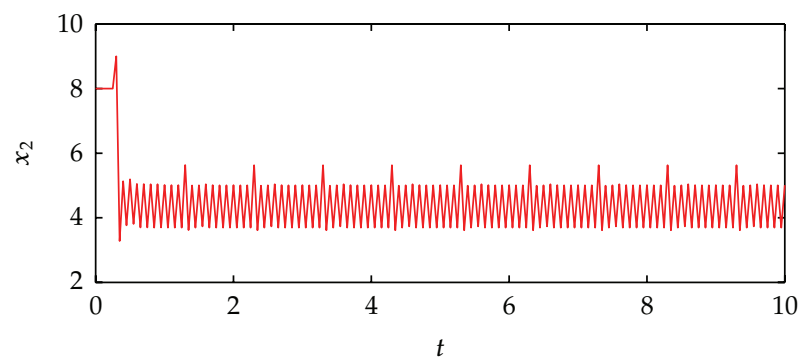

(b)

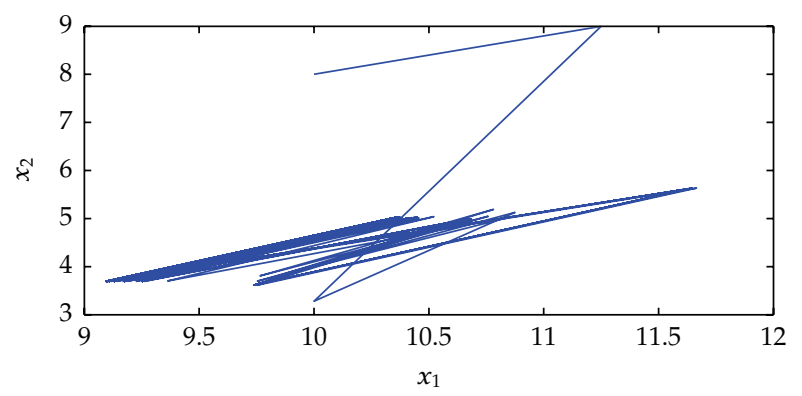

(c)

Figure 1: Dynamics of (4.2) - (a) time series of $x_{1}$, (b) time series of $x_{2}$, and (c) portrait of $\left(x_{1}, x_{2}\right)$.

of [1] fail, then one cannot obtain the existence of periodic solution of system (4.3) by results of reference [1], which further shows that the results complement or improve previously known results.

\section{Conclusions}

In this paper, the existence and globally exponential stability of the periodic solution of system (1.2) are studied. Model (1.2) is very general, including such models as continuous bidirectional associative memory networks, cellular neural networks, and Hopfield-type neural networks (see, e.g., $[6,7,28]$ ). The main methods employed here are Schaeffer' theorem, differential inequality techniques, and Lyapunov functional, which are very different from [1]. The sufficient conditions obtained here are new and complement or improve 


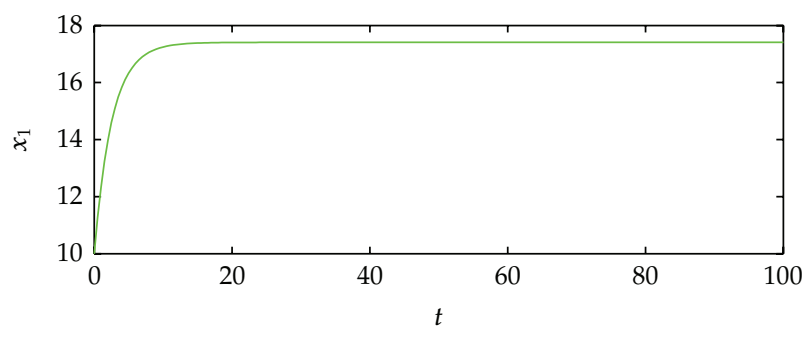

(a)

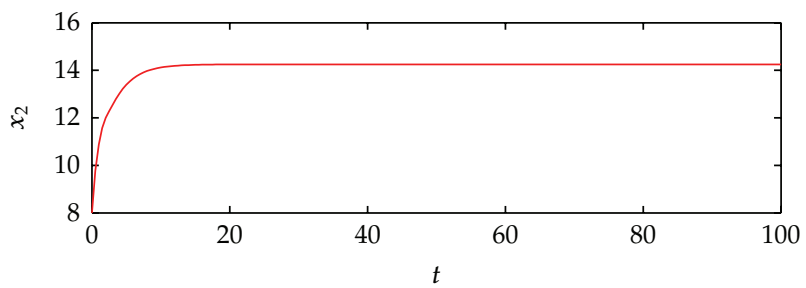

(b)

Figure 2: Dynamics of (4.3)—(a) time series of $x_{1},(\mathrm{~b})$ time series of $x_{2}$.

the previously known results [1,5-7]. Finally, applications, two illustrative examples and simulations, are given to show the effectiveness of the main results.

\section{Acknowledgments}

The authors would like to thank the reviewers for their valuable comments and constructive suggestions, which are very useful for improving the quality of this paper. This paper is supported by National Natural Science Foundation of China (11161015) and Doctoral Foundation of Guilin University of Technology (2010).

\section{References}

[1] M. Tan and Y. Tan, "Global exponential stability of periodic solution of neural network with variable coefficients and time-varying delays," Applied Mathematical Modelling, vol. 33, no. 1, pp. 373-385, 2009.

[2] $\mathrm{R}$. Wu, "Exponential convergence of BAM neural networks with time-varying coefficients and distributed delays," Nonlinear Analysis: Real World Applications, vol. 11, no. 1, pp. 562-573, 2010.

[3] I. A. Dzhalladova, J. Baštinec, J. Diblík, and D. Y. Khusainov, "Estimates of exponential stability for solutions of stochastic control systems with delay," Abstract and Applied Analysis, vol. 2011, Article ID 920412, 14 pages, 2011.

[4] K. Gopalsamy and Sariyasa, "Time delays and stimulus-dependent pattern formation in periodic environments in isolated neurons," IEEE Transactions on Neural Networks, vol. 13, no. 3, pp. 551-563, 2002.

[5] L. Huang, C. Huang, and B. Liu, "Dynamics of a class of cellular neural networks with time-varying delays," Physics Letters, Section A, vol. 345, no. 4-6, pp. 330-344, 2005.

[6] Y. Li, L. Zhu, and P. Liu, "Existence and stability of periodic solutions of delayed cellular neural networks," Nonlinear Analysis: Real World Applications, vol. 7, no. 2, pp. 225-234, 2006.

[7] B. Liu and L. Huang, "Existence and exponential stability of periodic solutions for cellular neural networks with time-varying delays," Physics Letters, Section A, vol. 349, no. 6, pp. 474-483, 2006. 
[8] Y. Shao and Y. Zhou, "Existence of an exponential periodic attractor of a class of impulsive differential equations with time-varying delays," Nonlinear Analysis: Theory, Methods E Applications, vol. 74, no. 4, pp. 1107-1118, 2011.

[9] C. Bai, "Global exponential stability and existence of periodic solution of Cohen-Grossberg type neural networks with delays and impulses," Nonlinear Analysis: Real World Applications, vol. 9, no. 3, pp. 747-761, 2008.

[10] Y. Shao and B. Dai, "The existence of exponential periodic attractor of impulsive BAM neural network with periodic coefficients and distributed delays," Neurocomputing, vol. 73, no. 16-18, pp. 3123-3131, 2010.

[11] Q. Zhou, "Global exponential stability of BAM neural networks with distributed delays and impulses," Nonlinear Analysis: Real World Applications, vol. 10, no. 1, pp. 144-153, 2009.

[12] Y. Xia and P. J. Y. Wong, "Global exponential stability of a class of retarded impulsive differential equations with applications," Chaos, Solitons and Fractals, vol. 39, no. 1, pp. 440-453, 2009.

[13] Y. Yang and J. Cao, "Stability and periodicity in delayed cellular neural networks with impulsive effects," Nonlinear Analysis: Real World Applications, vol. 8, no. 1, pp. 362-374, 2007.

[14] X. Li, X. Zhang, and D. Jiang, "A new existence theory for positive periodic solutions to functional differential equations with impulse effects," Computers $\mathcal{E}$ Mathematics with Applications, vol. 51, no. 12, pp. 1761-1772, 2006.

[15] J. J. Nieto, "Impulsive resonance periodic problems of first order," Applied Mathematics Letters, vol. 15, no. 4, pp. 489-493, 2002.

[16] X. Yang, "Existence and global exponential stability of periodic solution for Cohen-Grossberg shunting inhibitory cellular neural networks with delays and impulses," Neurocomputing, vol. 72, no. 10-12, pp. 2219-2226, 2009.

[17] J. Diblík, D. Ya. Khusainov, I. V. Grytsay, and Z. Šmarda, “Stability of nonlinear autonomous quadratic discrete systems in the critical case," Discrete Dynamics in Nature and Society, vol. 2010, Article ID 539087, 23 pages, 2010.

[18] R. Sakthivel, R. Samidurai, and S. M. Anthoni, "Asymptotic stability of stochastic delayed recurrent neural networks with impulsive effects," Journal of Optimization Theory and Applications, vol. 147, no. 3, pp. 583-596, 2010.

[19] R. Sakthivel, R. Samidurai, and S. M. Anthoni, "Exponential stability for stochastic neural networks of neural type with impulsive effects," Modern Physics Letters B, vol. 24, no. 11, pp. 1099-1110, 2010.

[20] J. Baštinec, J. Diblík, D. Ya. Khusainov, and A. Ryvolová, "Exponential stability and estimation of solutions of linear differential systems of neutral type with constant coefficients," Boundary Value Problems, vol. 2010, Article ID 956121, 20 pages, 2010.

[21] R. Samidurai, R. Sakthivel, and S. M. Anthoni, "Global asymptotic stability of BAM neural networks with mixed delays and impulses," Applied Mathematics and Computation, vol. 212, no. 1, pp. 113-119, 2009.

[22] R. Raja, R. Sakthivel, S. M. Anthoni, and H. Kim, "Stability of impulsive Hopfield neural networks with Markovian switching and time-varying delays," International Journal of Applied Mathematics and Computer Science, vol. 21, no. 1, pp. 127-135, 2011.

[23] Y. Shao, "Exponential stability of periodic neural networks with impulsive effects and time-varying delays," Applied Mathematics and Computation, vol. 217, no. 16, pp. 6893-6899, 2011.

[24] J. Chen and B. Cui, "Impulsive effects on global asymptotic stability of delay BAM neural networks," Chaos, Solitons and Fractals, vol. 38, no. 4, pp. 1115-1125, 2008.

[25] H. Wu and C. Shan, "Stability analysis for periodic solution of BAM neural networks with discontinuous neuron activations and impulses," Applied Mathematical Modelling, vol. 33, no. 6, pp. 2564-2574, 2009.

[26] D. Bainov and P. Simeonov, Impulsive Defferential Equatios: Periodic Solution and Applications, Pitman Monographs and Surveys in Pure and Applied Mathematics, Vol. 66, 1993.

[27] D. R. Smart, Fixed Point Theorems, Cambridge University Press, London, UK, 1974.

[28] S. Mohamad and K. Gopalsamy, "Dynamics of a class of discrete-time neural networks and their continuous-time counterparts," Mathematics and Computers in Simulation, vol. 53, no. 1-2, pp. 1-39, 2000. 


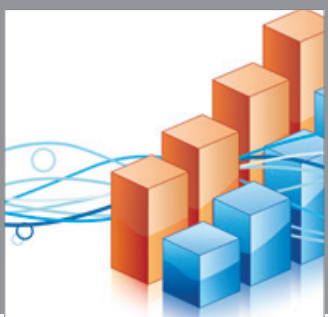

Advances in

Operations Research

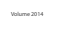

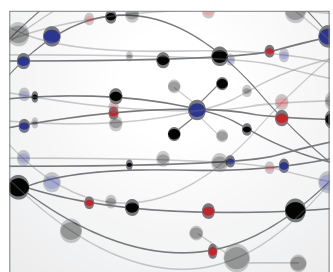

\section{The Scientific} World Journal
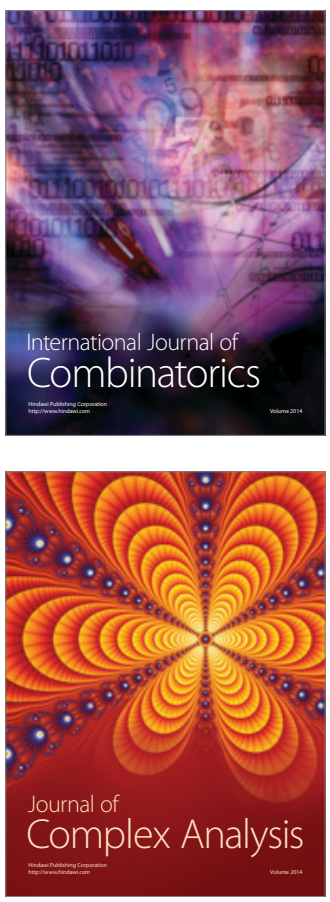

International Journal of

Mathematics and

Mathematical

Sciences
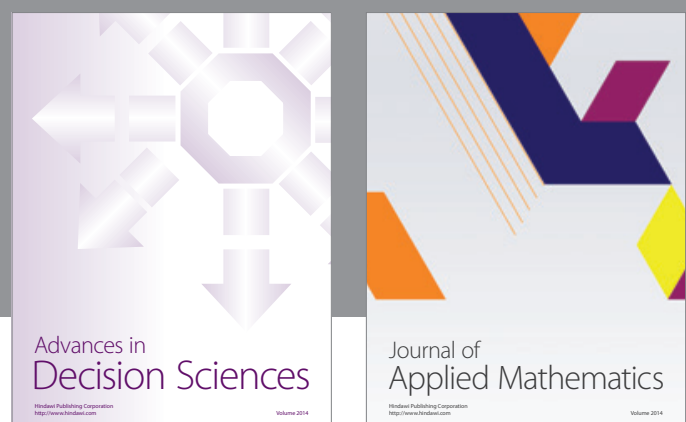

Journal of

Applied Mathematics
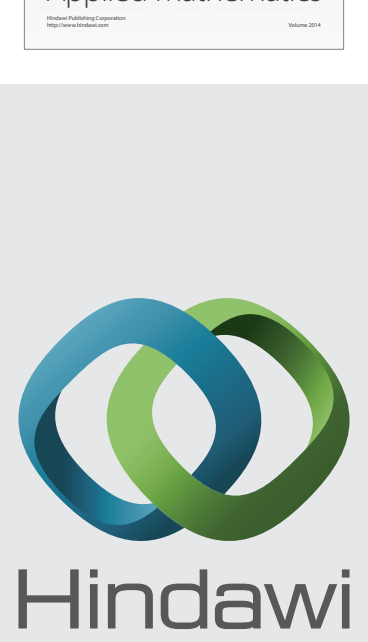

Submit your manuscripts at http://www.hindawi.com
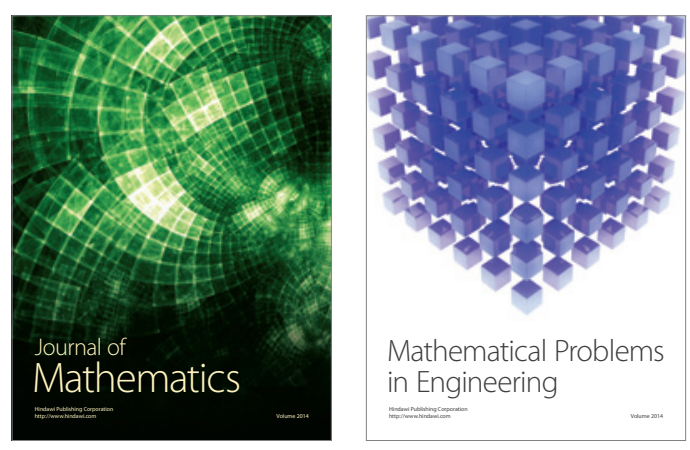

Mathematical Problems in Engineering
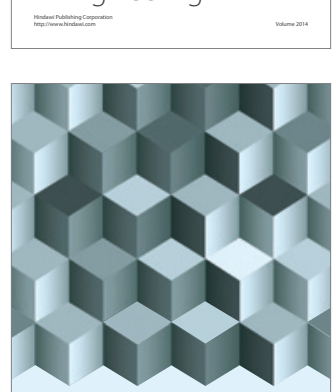

Journal of

Function Spaces
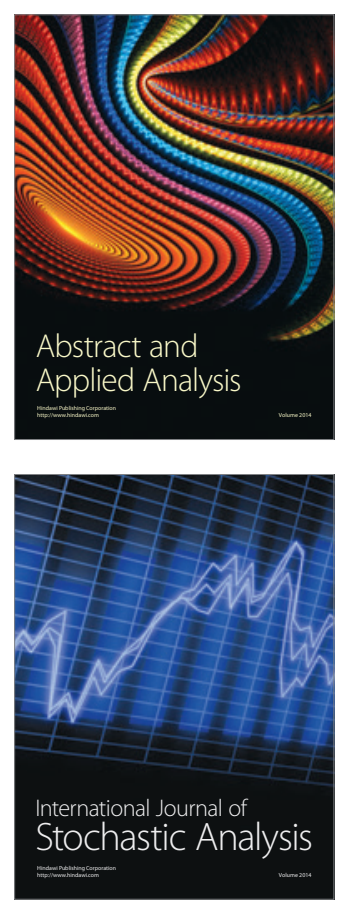

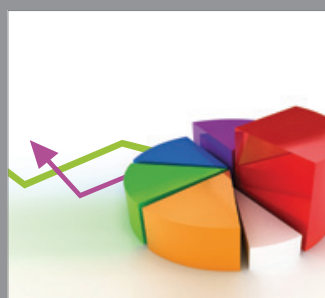

ournal of

Probability and Statistics

Promensencen
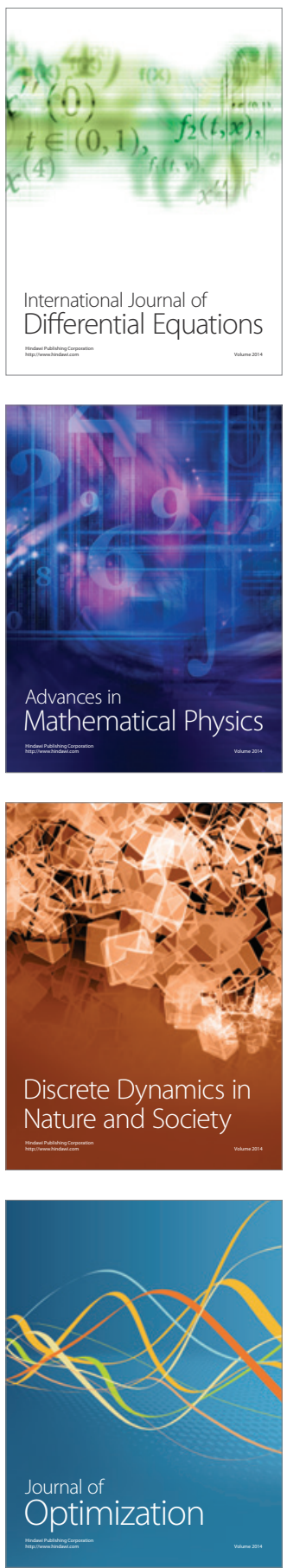